\title{
A Review on Various Clustering Technique and Protocol in MANET
}

\author{
Pooja Kumari ${ }^{1}$, Gurpreet Kaur ${ }^{2}$ \\ M.Tech Student, Computer Science, Global Institute of Management \& Emerging Technologies, PTU Jalandhar, Punjab ${ }^{1}$ \\ Asst. Prof, Computer Science, Global Institute of Management \& Emerging Technologies, PTU Jalandhar, Punjab ${ }^{2}$
}

\begin{abstract}
Mobile ad-hoc network (MANETs) is a type of wireless network which do not consist a base station for providing network connectivity. A mobile ad-hoc network (MANET) is a self-configuring, infrastructure less network. When MANET network used for large scale operation, MANET induce many drawbacks like delay in routing, resource consumption due to dynamic nature of MANET. Many cluster algorithm proposed by researcher to overcome this problem. Cluster technique used to splitting the network into Sub network called cluster. Every cluster has a its own cluster head which maintains a whole information about the cluster. Dynamic nature of MANET make difficult to divide a network into cluster and electing cluster head. In this paper we provide an overview of some clustering approaches and protocol used in Manet.
\end{abstract}

Keyword: MANET, Cluster, Clustering Protocol.

\section{INTRODUCTION}

A mobile ad hoc network (MANET) is a self-configurable infrastructure less network of mobile nodes connected by wireless link. Ad hoc[1] network do not rely on any fixed infrastructure(infrastructure based network nodes are connected to fixed base station). In MANET communication is done by using multihop transmission. In multihop[2] transmission if two node are not in the range than another intermediate connected nodes are used to make transmission possible. In mobile adhoc network mobile nodes are free to move anywhere in the network so it leads to distortion in communication in link. In mobile adhoc[3] network nodes not only behave as host but also as a router for providing route.

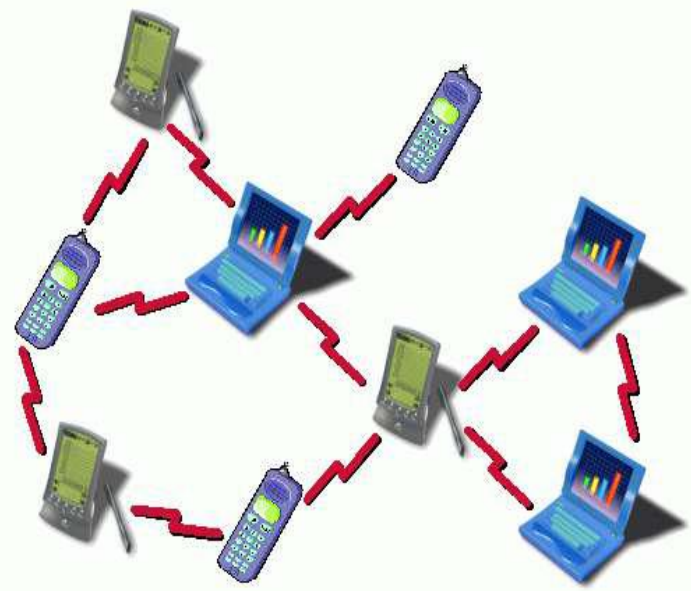

Fig 1 Mobile Adhoc network

\section{Advantages of MANET}

MANET has following advantages over mobile network with fixed infrastructure.

1) Autonomous behaviour- In MANET, each node not only behave as host but also router[4] which means that a node can be host and can also perform routing operations as router.

2) Multi-hop transmission- Manet uses a multihop transmission when source node and destination node is out of transmission range.

3) Distributed nature of operation- There is no centralized control mechanism, operations of network distributed among nodes.

4) Dynamically changing topology- MANET is dynamic topology network, nodes can join and leave network anytime.

5) Infrastructure less network-Mobile Adhoc network do not rely on any fixed infrastructure. 


\section{Applications of MANET}

1) Military battlefield- Military equipment[5] now routinely contains some sort of computer Equipment to maintain an information network among the vehicles, soldiers and military head quarters.

2) Commercial sector- Ad hoc network can be used in emergency/rescue operations for natural tragedy relief efforts, e.g. in fire, flood, or earthquake.

3) Local level- Ad hoc networks can make free temporary link multimedia network using palmtop computers or notebook computers to distribute and share data among participants e.g. conference.

4) Personal Area Network - Traditional wired cables are replaced with wireless connections. MANET can also expand to access the Internet or other networks by using mechanisms e.g. Wireless LAN.

\section{(ii) Clustering Overview}

A Clustering is[6] a method of splitting a network into sub network called cluster. Each and every cluster has a its own cluster head which maintains a whole information about the cluster.

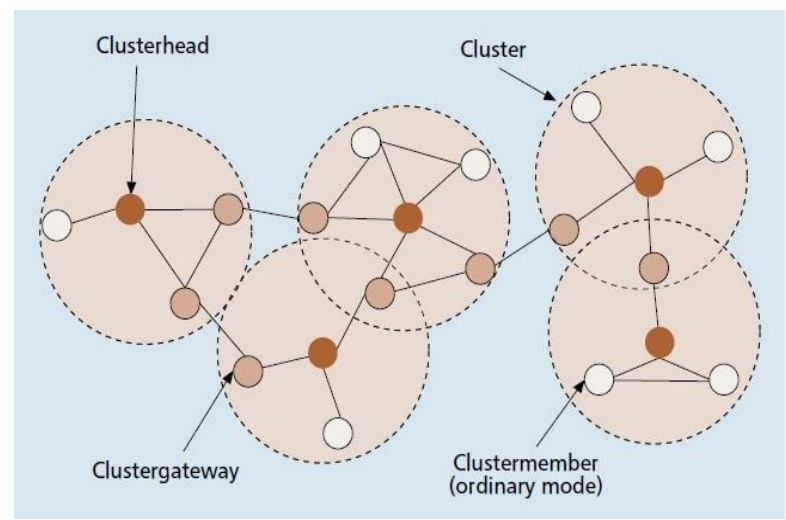

Fig 2 Cluster Structure

Cluster Structure includes three types of nodes:-

Cluster head: - Every cluster[7] has a cluster head which coordinates all the nodes within the cluster. Various techniques are introduce for electing a cluster head in cluster.

Cluster member: - Normal nodes that transmits information to their cluster head which compresses this information and forward it to other cluster head.

Cluster gateway node:- non cluster head node that is employed for making interaction among different clusters, forward information between clusters.

\section{(iii) CLUSTERING PROTOCOL}

Routing techniques are classified into various categories like location based, mobility based, multipath based etc. In this paper, some clustering protocols are discussed.

1. Low Energy Adaptive Clustering Hierarchy (LEACH):- Leach is the first [8] proposed clustering protocol. The main goal of this protocol is to improve the lifetime of network by equally distributing the energy consumption among the all nodes and reducing the energy consumption by making a data aggregation. In leach, load balancing done by cluster head in cluster.

2. PEGASIS: - PEGASIS stands for power efficient gathering in sensor information system. This protocol creates a chain among nodes. Data pass through node to node. New chain will be reconstructed whenever one node dies in the network. This approach will distribute the energy equally load among the sensor nodes within the network. If the chain is created by the nodes themselves, they will first get the information of the location of all nodes and locally compute the chain using the greedy algorithm.

3. Unequally Clustered Multihop Routing (UCMR) protocol:- Most of the clustering algorithm provides an equal cluster size. In a Unequally Clustered [8] Multihop Routing (UCMR) protocol is given where every cluster incorporates a different cluster size, based on its distance with reference to base station. When distance from base station increases .The size of the cluster is also increasing.

4. Clustering Algorithm via Waiting Timer (CAWT):- CAWT is a distributed proximity-connectivity-based algorithm for making cluster. Once nodes are deployed in network, each node sends a "hello" message to the neighbour node to show the existence. The node that has number of "hello" messages organize into clusters while others are waiting to form clusters. 


\section{(iv) CLUSTER FORMATION ALGORITHM}

\section{Direct trust evaluation technique}

In this technique [9] trust value for each node is calculated for selecting a cluster head in cluster. This trust value is evaluated based on some matrices like type of data forwarded, no of packet forwarded, delivery rate, dropped etc. This trust value is than compare with predefined threshold value for electing cluster head.

Cluster based route discovery algorithm(AODV)

This algorithm concentrate [10] on two conditions in clustered network. First intra cluster route discovery (sender node and receiver node presents in the same cluster). Second inter cluster (sender and receiver node presents in the different cluster) the algorithm uses the concept of history table used to store the history of previous transfers. This will help in saving a time and resources energy.

Connectivity Based Clustering:- Cluster head is elected based on the connectivity. The node with higher no of neighboring node considered as cluster head itself and all other neighboring join the cluster as cluster member.

Energy Efficient Clustering (FWCABP):-FWCABP stands[11] for Flexible Weighted Clustering Algorithm based on Battery Power. This clustering algorithm is based on battery power of nodes. Nodes with minimum energy power are preventing for becoming a cluster head. In the cluster formation phase every node sends a massage to neighboring node to inform its status and to build a neighbor list. Node elected as cluster head based on matrices:- weight of the degree of nodes, nodes mobility, nodes remaining battery power and sum of distance to its neighboring nodes. The node with minimum weight will be selected as cluster head. Cluster maintenance phase implement when node goes outside cluster region or when battery power of cluster head decreases to a predefined threshold value.

\section{CONCLUSION}

This review paper represent a MANET and clustering includes definition of Mobile Adhoc Network, Advantages of Manet, Application of manet, overview of clustering, clustering protocol, cluster formation technique. Clustering methods improve network scalability, routing, and minimize network overhead.

\section{REFERENCES}

[1] NehaSingla, Ruby Gupta" A Review of Performance Evaluation of the Routing Protocols in MANETs" Vol. 2, Issue 11, November 2014.

[2] Vaibhav V. Bhujade, Deepak Chaudhary, Suraj V. Raut" A Review Paper on Behavior of Node in MANET" International Journal on Recent and Innovation Trends in Computing and Communication ISSN: 2321-8169 Volume: 3 Issue: 4.

[3] Ms. Aastha kohli , Mr. Sukhbir "A Review paper on Routing Protocol Comparison" Vol. 1 Issue II, September2013 ISSN: $2321-9653$.

[4]"http://www.eexploria.com/manetmobile-ad-hoc-network-characteristics-and-features/"

[5] Jun-Zhao Sun "Mobile Ad Hoc Networking: An Essential Technology for Pervasive Computing" 0.7803 -70 10-4/0 1/S 10.000200 1 IEEE.

[6] Ipsita Panda “A Clustering approach in Mobile Ad-hoc Networks Routing” International Journal of Computer Science \& Engineering Technology (IJCSET) Vol. 4 No. 03 Mar 2013.

[7] Ankita Singh Kushwah, Nitin Manjhi “A Review of Cluster Formation Schemes in MANET” International Journal of Application or Innovation in Engineering \& Management (IJAIEM) Volume 3, Issue 4, April 2014.

[8] Harpinder Kaur, Navjot kaur, Sandeep Waraich “ Comparative Analysis of Clustering Protocols for Wireless Sensor Networks" International Journal of Computer Applications (0975 - 8887) Volume 115 - No. 1, April 2015.

[9] K.Gomathi, B.Parvathavarthini "An Efficient Cluster based Key Management Scheme for MANET with Authentication" 978-1-4244-90080/10/\$26.00 c2010 IEEE.

[10] Poonam Thakur, Dr. Anita Ganpati "Cluster Based Route Discovery Technique for Routing Protocol in MANET" 2015 International Conference on Green Computing and Internet of Things (ICGCloT) 623.

[11] Amit Savyanavar, Mohini Borate "Survey of Clustering Schemes in Mobile Ad hoc Networks" International Journal of Science and Research (IJSR) Volume 3 Issue 11, November 2014. 